Research Paper

\title{
Baseline Metabolic Risk Score and Postsurgical Esophageal Cancer-Specific Mortality: The Fujian Prospective Investigation of Cancer (FIESTA) Study
}

\author{
Hong Sha ${ }^{1^{*}}$, Dan $\mathrm{Hu}^{2^{*}}$, Sinan $\mathrm{Wu}^{1^{*}}$, Feng Peng ${ }^{3{ }^{凶 *}}$, Guodong $\mathrm{Xu}^{1}$, Guohui Fan ${ }^{1}$, Xiandong Lin ${ }^{2}$, Gang Chen ${ }^{2}$, \\ Binying Liang ${ }^{4}$, Ying Chen ${ }^{5}$, Chao $\mathrm{Li}^{2}$, Hejun Zhang ${ }^{2}$, Yan Xia ${ }^{2}$, Jinxiu Lin ${ }^{3}$, Xiongwei Zheng ${ }^{2}$, Wenquan \\ $\mathrm{Niu}^{1 凶}$ \\ 1. Institute of Clinical Medical Sciences, China-Japan Friendship Hospital, Beijing, China. \\ 2. Department of Pathology, Fujian Cancer Hospital and Fujian Medical University Cancer Hospital, Fuzhou, Fujian, China. \\ 3. Department of Cardiology, The First Affiliated Hospital of Fujian Medical University, Fuzhou, Fujian, China. \\ 4. Department of Medical Record, Fujian Cancer Hospital and Fujian Medical University Cancer Hospital, Fuzhou, Fujian, China. \\ 5. Department of Core Research Laboratory, Fujian Cancer Hospital and Fujian Medical University Cancer Hospital, Fuzhou, Fujian, China. \\ *Shared first authors. \\ $\triangle$ Corresponding author: Wenquan Niu, Ph.D. Address: No. 2 Yinghuayuan East Street, Chaoyang District, Beijing 100029, China. Tel \& Fax: 86-01-8420 6414. \\ E-mail: niuwenquan_shen@163.com or niuwenquan@zryhyy.com.cn or Xiongwei Zheng, M.D. Ph.D. Address: Fu Ma Road 420, Jin An District, Fuzhou 350014, \\ Fujian, China. Phone: +86-591-8364 3149. Fax: +86-591-8392 8767. E-mail: agu1960@126.com or Feng Peng, M.D. Ph.D. Address: Cha Zhong Road 20, Tai Jiang \\ District, Fuzhou 350005, Fujian, China. Phone: +86-591-8798 1637. Fax: +86-591-8798 1635. Email: pengfeng@medmail.com.cn. \\ (c) Ivyspring International Publisher. This is an open access article distributed under the terms of the Creative Commons Attribution (CC BY-NC) license \\ (https://creativecommons.org/licenses/by-nc/4.0/). See http://ivyspring.com/terms for full terms and conditions.
}

Received: 2017.11.02; Accepted: 2017.12.28; Published: 2018.03.08

\begin{abstract}
Backgrounds: Compelling evidence has emerged to support a close relationship between metabolic syndrome and esophageal cancer (EC).

Aims: Using five baseline metabolism-related markers, we constructed a metabolic risk score (MRS), aiming to test whether MRS can improve the prediction of postsurgical EC-specific mortality over traditional demographic and clinicopathologic characteristics.

Methods: Total 2535 EC patients who received three-field lymphadenectomy were enrolled from January 2000 to December 2010, and they were followed up until December 2015.

Results: All EC patients were randomly split into derivation group $(n=1512,60 \%)$ and validation group $(n=1014,40 \%)$. MRS was generated in derivation group by adopting the Framingham 'points' system and shrinkage method, and it ranged from -9 to 17. EC-specific mortality risk increased with the increase of MRS, and adjusted estimates were more obvious in patients with upper tertile (MRS>6) than patients with lower MRS $(\leq 2)$ in either derivation (hazard ratio $[H R]=2.28,95 \%$ confidence interval $[\mathrm{Cl}]: 1.90-2.73, \mathrm{P}<0.001)$ or validation group $(\mathrm{HR}=2.11,95 \% \mathrm{Cl}$ : 1.66-2.67, $\mathrm{P}<0.001)$ or both groups $(\mathrm{HR}=2.37,95 \% \mathrm{Cl}: 1.95-2.88, \mathrm{P}<0.001)$. In Kaplan-Meier curve, cumulative survival rates differed significantly across tertiles of MRS. Further analysis indicated that MRS can improve classification accuracy and discriminatory ability over clinicopathologic parameters.

Conclusions: Our findings supported the usefulness of baseline MRS in predicting the prognosis of postsurgical EC-specific mortality.
\end{abstract}

Key words: Esophageal cancer; The FIESTA study; Metabolic risk score; Prognosis.

\section{Introduction}

Esophageal cancer (EC) is among the top five most prevalent types of cancer in China, with the EC toll rising to an estimated 477,900 new cases and
375,000 deaths in 2015. There are two major types of EC: esophageal squamous-cell carcinoma (ESCC) and esophageal adenocarcinoma (EAC), and their 
prevalence varies widely across countries [1]. In China, the most prevalent type is ESCC, which accounts for upwards of 90 percent of EC cases, while the incidence of EAC by contrast is progressively increasing in the U.S. during recent decades $[2,3]$. Early EC is asymptomatic, and it is often diagnosed at an advanced stage. Epidemiological studies have shown that EC is a devastating disease and has a poor prognosis, with the 5-year survival rate of approximately $20 \%$ in China [4,5]. Despite decades of achievements in cancer management and introduction of numerous candidate or putative markers, improvements in the diagnosis and prognosis of EC are still unsatisfactory. Due to complexity of esophageal carcinogenesis, it is a reasonable proposal to adopt a panel of markers for early detection and targeted intervention to reduce escalating burden of EC worldwide. Therefore, the adoption of risk prediction models for the development of EC will no doubt improve its prognosis.

Compelling evidence has emerged to support a close relationship between metabolic syndrome and cancer risk [6-8]. Metabolic syndrome represents a clustering of cardiovascular risk factors, including obesity, dysglycemia, elevated blood pressure, high circulating triglyceride (TG) and low circulating high-density lipoprotein cholesterol (HDLC). More recently, we in an ongoing prospective cohort study the Fujian prospective investigation of cancer (FIESTA) study [9-15], have assessed the prediction of baseline metabolic syndrome and its components for cancer specific mortality of common digestive malignancies. Importantly, our findings have demonstrated that the concomitance of baseline metabolic syndrome was identified as a significant independent predictor for high ESCC-specific mortality after three-field lymphadenectomy. As an essential and necessary supplement to our previous findings and in light of carcinogenic potentials of metabolic syndrome, we constructed a metabolic risk score (MRS) using five baseline metabolism-related markers by adopting the methodology of the Framingham coronary heart disease prediction model [16], aiming to test whether MRS can improve the prediction of postsurgical EC-specific mortality over traditional demographic and clinicopathologic characteristics.

\section{Methods}

\section{Study patients}

As a part of FIESTA study [9-15], the current study involved 2886 EC patients who were consecutively admitted at Department of Thoracic Surgery, Fujian Cancer Hospital to receive three-field lymphadenectomy for the first time, and were safely discharged after the surgery during the period between January 2000 and December 2010. At the time of recruitment, all patients gave written informed consent for use of blood and tissue samples, as well as subsequent analyses. Meanwhile, they completed questionnaires on demographic and anthropometric characteristics, including birthdate, age at the surgery for EC, gender, smoking, drinking and family cancer history. Ethical approval was obtained from the ethics committee of Fujian Cancer Hospital (No. SQ2015-070-01).

\section{Diagnosis}

EC diagnosis was confirmed by presurgical biopsies or postsurgical pathologic analyses. Moreover, all study patients received no presurgical and postsurgical chemotherapy or radiotherapy.

\section{Blood and tissue samples}

Fasting venous blood samples were drawn at the day before receiving three-field lymphadenectomy for EC when patients were fasted overnight. During the surgery, paired cancerous tissue and normal esophagus tissue were excised from each patient, and they were fixed in 10\% neutral-buffered formalin and paraffin-embedded using standard procedures.

Fasting blood samples were used to measure blood glucose, TG, total cholesterol (TC), HDLC and low-density lipoprotein cholesterol (LDLC). All measurements were done according to the same standard procedures by qualified operators at the same hospital. Tissue samples were used for clinicopathological analysis at the Department of Pathology of Fujian Cancer Hospital.

\section{Demographic measurement}

Body weight and height were measured at the time of admission when study subjects wore light clothes and were barefooted. Body mass index (BMI) was computed as the weight (kilogram)/height (meter) ${ }^{2}$. Arterial systolic/diastolic blood pressure (SBP/DBP) was measured with conventional mercury sphygmomanometer on three occasions of at least 5 min intervals by certified examiners. Smoking was grouped into ever (former or current) smoking and never smoking. Drinking was grouped into ever (former or current) drinking and never drinking. A patient was recorded to have a positive family cancer history if one or more of his/her affected relatives within three generations had suffered any malignances besides the non-melanoma skin cancer.

\section{Clinicopathologic evaluation}

After the surgery, clinicopathologic data were abstracted from pathological reports, including 
histological type of EC (ESCC, EAC and esophageal neuroendocrine carcinomas), tumor size, tumour node metastasis (TNM) stage (I, II, III and IV per the $7^{\text {th }}$ Edition of the UICC/AJCC TNM Staging System [17]), depth of invasion (T1-T4), regional lymph node metastasis (LNM) (N0-N3), distant metastasis (M0 and M1), tumor location (upper, middle and lower esophagus), histological differentiation (well, moderate and poor differentiation) and tumor embolus.

\section{Metabolic risk score}

According to diagnostic criteria proposed by the Chinese Diabetes Society in 2004 [18], metabolic syndrome is defined by the coexistence of three or more of the following symptoms: obesity (BMI $\geq 25$ $\mathrm{kg} / \mathrm{m}^{2}$ ), hyperglycemia (fasting glucose $\geq 6.1 \mathrm{mmol} / \mathrm{L}$ or 2-hour plasma glucose $\geq 7.8 \mathrm{mmol} / \mathrm{L}$ or previously diagnosed diabetes), hypertension (SBP/DBP $\geq$ $140 / 90 \mathrm{mmHg}$ or under antihypertensive therapy) and dyslipidemia (TG $\geq 1.7 \mathrm{mmol} / \mathrm{L}$ or HDLC $<0.9$ $\mathrm{mmol} / \mathrm{L}$ in men or $<1.0 \mathrm{mmol} / \mathrm{L}$ in women). Considering the close relevance between SBP and $\mathrm{DBP}$, pulse pressure (PP), the difference between SBP and DBP, was calculated accordingly. As these factors of metabolic origin tend to occur together, MRS was hence generated based on baseline BMI, PP, fasting glucose, TG and HDLC.

As far as we know, the Framingham heart study has led the development and dissemination of multivariable statistical models to estimate the risk of coronary heart disease. The Framingham heart study has introduced a 'points' system that can construct complex statistical models more practical for clinicians [19]. Given the overestimating risk of multivariate regression model associating metabolismrelated factors with postsurgical mortality, shrinkage method was adopted to abate this risk [20]. In this present study, we employed the rationales of 'points' system and the validity of shrinkage method to generate MRS when all five metabolism-related factors were analyzed in quintiles, and the detailed process is illustrated in Supplementary Table 1.

\section{Follow-up assessment}

After discharge from the hospital, all patients were followed up every six to twelve months at the Out-Patient Department of Fujian Cancer Hospital or via phone calls or postal letters if the patients missed appointments or failed to appear. Death from EC was defined as the clinical event of concern. The time to event was calculated in months from the date of surgery to death date or date of the last follow-up, whichever occurred first. The minimum postsurgical 5-year follow-up for study patients provided us the ability to make predictions at the 5-year time point.

Prior to December 2015, approximately 5\% $(n=147)$ and $7 \% \quad(n=204)$ of study patients after discharge were lost to follow-up and died from causes other than EC, respectively. The baseline and clinicopathologic characteristics of these 351 patients are presented in Supplementary Table 2. Consequently, 2535 patients with complete survival data were eligible in the final analysis, and age range was 30-88 years. At the end the latest follow-up in 2015, 1265 deaths from EC occurred, leaving 1270 survivors. The 5-year survival rate of 2535 patients was $52.2 \%$, comparable with the corresponding number reported in previous studies [21,22].

\section{Statistical analysis}

According to previous experience [23], we developed MRS in a randomly selected $60 \%$ of study patients (derivation group: $\mathrm{n}=1512$ ), and validated it in the rest $40 \%$ patients (validation group: $n=1014$ ). The demographic and clinicopathologic characteristics were compared between derivation and validation groups by using unpaired t-test or Mann-Whitney U-test or $\chi^{2}$-test as appropriate. Based on distributions, aforementioned five metabolismrelated factors were categorized into quintiles. Crude and adjusted risk estimates of derived MRS, both in isolation and in tertiles, for EC-specific mortality were quantified using the multivariate Weibull proportional hazards regression model, mainly because EC mortality rate exhibited a smooth increasing tendency over the annual follow-up from the year 2000 to 2015 and from the statistical aspect that the $\ln (-\ln (S(t)))$ was a linear function of $\ln (t)$ (Here, $t$ denotes survival time and $S(t)$ denotes survival function). Kaplan-Meier curve was plotted according to the tertiles of derived MRS, and cumulative survival rates were compared with Log-rank test. Median survival time was calculated by tertile classification of derived MRS.

Clinical usefulness of derived MRS was evaluated by using decision curve analysis [24]. Decision curve analysis is methodologically based on the prediction of modified risk model over original model. Decision curve analysis was illustrated as a graph, with the $\mathrm{x}$-axis denoting thresholds for EC-specific mortality and the y-axis denoting net benefit for different thresholds. A higher net benefit is provided by these prediction models that are farthest away from solid curve line when assuming all EC-specific mortality and dotted horizontal line when assuming none EC-specific mortality.

The added usefulness (i.e. predictive accuracy for EC-specific mortality) of derived MRS to the widely-accepted TNM staging system was assessed 
from both discrimination and calibration aspects. With regard to discrimination, both Harrell C-statistic and area under the receiver operating characteristics (ROC) curves were calculated to inspect whether derived MRS had an ability to differentiate among patients who died of EC or survived. Areas under the ROC curve were compared accordingly between models with and without derived MRS. For calibration, $-2 \log$ likelihood ratio test, as well as Akaike information criterion (AIC) and Bayesian information criterion (BIC) were performed to assess how closely the predictive probabilities of estimated risk for EC-specific mortality by adding derived MRS reflected actual observed risk, as well as global fit of models.

Data were managed by using STATA/SE software Release 14.1 (StataCorp, TX, USA) on the Macintosh. A significance level of $\mathrm{P}<0.05$ was used unless otherwise stated, and all tests were two-sided.

\section{Results}

\section{Comparison of derivation and validation groups}

Total 2535 postsurgical EC patients were randomly split into the derivation and validation groups, incorporating 1512 (60\%) and 1014 (40\%) patients, respectively. The demographic and clinicopathologic characteristics of the two groups are compared in Table 1. Except for esophagus location with marginal difference $(\mathrm{P}=0.059)$, all demographic and clinicopathologic characteristics were perfectly comparable between the two groups. Median survival time was 92.2 months in derivation group and 113.3 months in validation group, the difference being nonsignificant $(\mathrm{P}=0.2411)$.

\section{Metabolic risk score}

Based on the Framingham 'points' system and shrinkage method, each of five baseline metabolism-related factors in derivation group was classified into quintiles and standardized into scores (Supplementary Table 1). For each patient, individual scores were summed to generate a total score value, termed as MRS, which ranged from -9 to 17 in derivation group. The frequency distributions of derived MRS are plotted in Figure 1.

\section{Prognostic prediction}

In view of the low frequencies of extreme scores, we combined patients with MRS equal to or less than 6 together as a single score group, as well as for MRS equal to or greater than 15 . The risk prediction of derived MRS, both in isolation and in tertiles, for EC-specific mortality is summarized in Table 2 and Table 3, respectively. In view of statistical power, individual assessment of derived MRS was implemented in all study patients with and without considering demographic and clinicopathologic characteristics.

Table 1. Comparison of baseline characteristics between patients in derivation and validation groups

\begin{tabular}{|c|c|c|c|}
\hline Characteristic & Derivation group & Validation group & $\mathbf{P}$ \\
\hline Number & 1512 & 1014 & \\
\hline Age (years) & $56.65(9.41)$ & $56.76(9.07)$ & 0.765 \\
\hline $\operatorname{Sex}(M)$ & $76.59 \%$ & $75.35 \%$ & 0.470 \\
\hline BMI $\left(\mathrm{kg} / \mathrm{m}^{2}\right)$ & $22.26(2.96)$ & $22.38(3.09)$ & 0.336 \\
\hline Ever smoking & $41.93 \%$ & $41.81 \%$ & 0.961 \\
\hline Ever drinking & $19.78 \%$ & $20.51 \%$ & 0.674 \\
\hline Family history (+) & $12.90 \%$ & $15.29 \%$ & 0.104 \\
\hline SBP (mmHg) & 123.80 (17.69) & 124.58 (19.16) & 0.292 \\
\hline DBP (mmHg) & 77.47 (10.42) & 77.51 (19.16) & 0.921 \\
\hline FBG (mmol/L) & $6.12(2.53)$ & $6.10(2.56)$ & 0.828 \\
\hline TG (mmol/L) & $1.19(0.87)$ & $1.20(0.94)$ & 0.755 \\
\hline $\mathrm{TC}(\mathrm{mmol} / \mathrm{L})$ & $4.84(1.04)$ & $4.83(1.04)$ & 0.852 \\
\hline HDLC (mmol/L) & $1.12(0.41)$ & $1.13(0.44)$ & 0.409 \\
\hline LDLC (mmol/L) & $3.19(0.94)$ & $3.19(0.93)$ & 0.999 \\
\hline Esophagus location & & & 0.059 \\
\hline Upper & $8.99 \%$ & $11.24 \%$ & \\
\hline Middle & $81.94 \%$ & $77.91 \%$ & \\
\hline Lower & $9.07 \%$ & $10.85 \%$ & \\
\hline Histological differentiation & & & 0.324 \\
\hline Well & $15.08 \%$ & $14.00 \%$ & \\
\hline Moderate & $63.76 \%$ & $66.67 \%$ & \\
\hline Poor & $21.16 \%$ & $19.33 \%$ & \\
\hline Depth of invasion & & & 0.301 \\
\hline $\mathrm{T} 1-\mathrm{T} 2$ & $28.70 \%$ & $28.50 \%$ & \\
\hline T3-T4 & $71.30 \%$ & $71.50 \%$ & \\
\hline Regional LNM & & & 0.414 \\
\hline N0 & $43.85 \%$ & $43.29 \%$ & \\
\hline N1 & $26.52 \%$ & $25.25 \%$ & \\
\hline N2 & $18.98 \%$ & $21.60 \%$ & \\
\hline N3 & $10.65 \%$ & $9.86 \%$ & \\
\hline TNM stage & & & 0.302 \\
\hline I & $8.66 \%$ & $10.45 \%$ & \\
\hline II & $32.87 \%$ & $32.15 \%$ & \\
\hline III & $58.47 \%$ & $57.40 \%$ & \\
\hline Tumor embolus (+) & $16.14 \%$ & $17.26 \%$ & 0.472 \\
\hline Tumor size $(\mathrm{cm})$ & $4.53(2.08)$ & $4.41(1.97)$ & 0.163 \\
\hline \multicolumn{4}{|c|}{$\begin{array}{l}\text { Abbreviations: BMI, body mass index; SBP, systolic blood pressure; DBP, diastolic } \\
\text { blood pressure; FBG, fasting blood glucose; TG, triglycerides; TC, total cholesterol; } \\
\text { HDLC, high-density lipoprotein cholesterol; LDLC, low-density lipoprotein } \\
\text { cholesterol; LNM, lymph node metastasis; TNM, tumour node metastasis. Data are } \\
\text { expressed as mean } \pm \text { standard deviation or percentage. P was calculated by the } \\
\text { t-test or the Mann-Whitney U test or the Chisq test, where appropriate. }\end{array}$} \\
\hline
\end{tabular}

As expected, there was a tendency of increase in the magnitude or risk prediction with the increase of MRS (Table 2). When MRS was grouped in tertiles, risk prediction was more obvious among patients with upper tertile (MSR>6) than patients with lower MRS $(\leq 2)$ in either derivation group $(\mathrm{HR}=2.28,95 \%$ CI: $1.90-2.73, \mathrm{P}<0.001)$ or validation group $(\mathrm{HR}=2.11$, 95\% CI: $1.66-2.67, \quad \mathrm{P}<0.001)$ or combined group (HR=2.37, 95\% CI: 1.95-2.88, $\mathrm{P}<0.001)$ after adjusting for demographic and clinicopathologic characteristics (Table 3). 


\section{Cumulative survival difference}

As illustrated in Figure 2, Kaplan-Meier curve was plotted by the tertiles of derived MRS in both derivation and validation groups. EC patients in middle and upper tertiles of derived MSR had significantly lower median survival time than those in lower tertile in both derivation (30 months and 77.1 months vs. 163 months) and validation (32.4 months and 113 months vs. 163 months) groups. Log-rank test revealed remarkable significance in both groups $(\mathrm{P}<0.0001)$.

\section{Decision curve analysis}

The added usefulness of derived MRS was evaluated graphically by decision curve analysis, as shown in Supplementary Figure 1. The net benefit for the addition of MRS over demographic and clinicopathologic characteristics was significant in both derivation and validation groups, justifying the practicality of derived MRS on clinical decisionmaking.
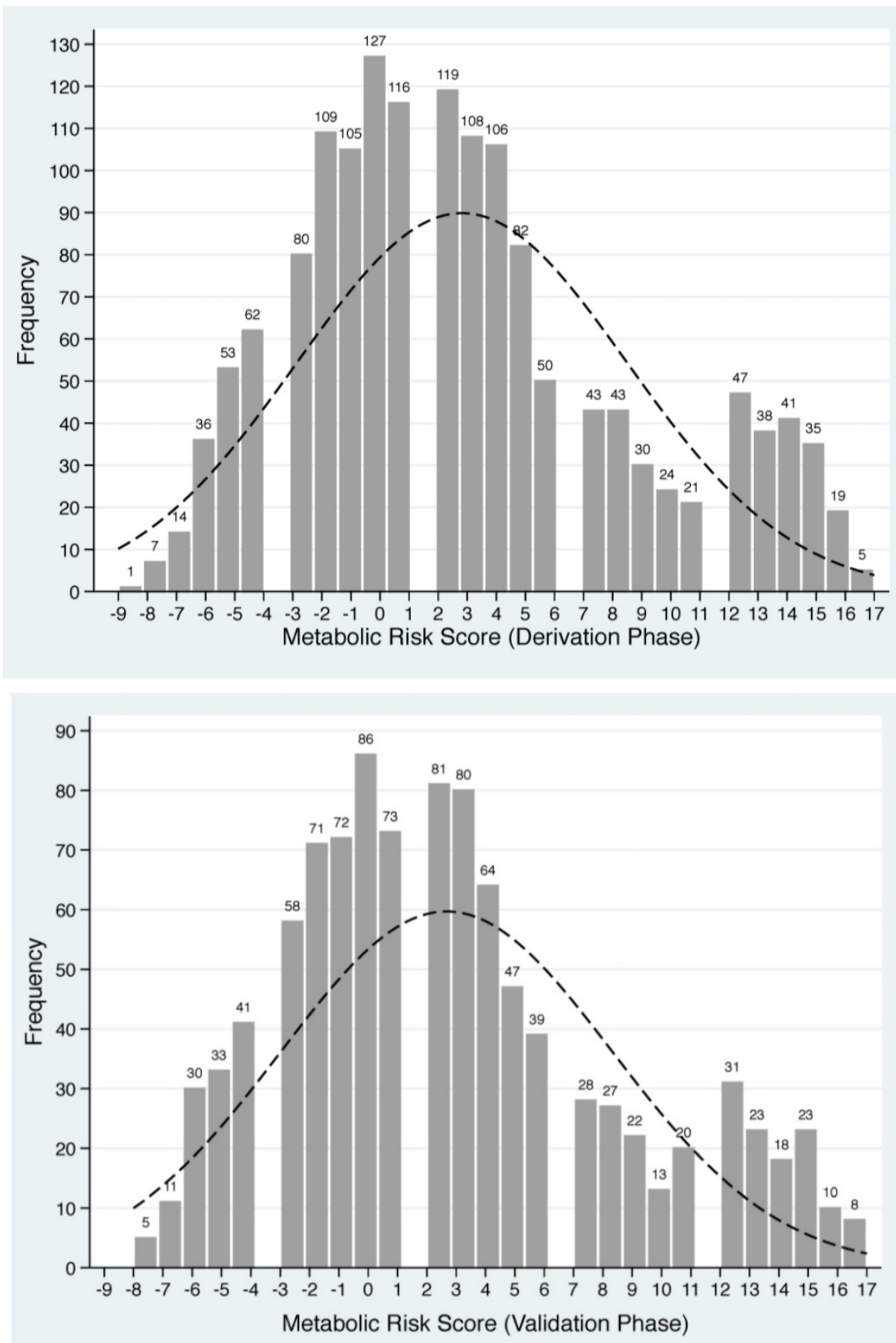

Figure 1. Distributions of derived metabolic risk score in derivation (the upper panel) and validation (the lower panel) groups. The number on the bar represents the count of individual metabolic risk score. 
Table 2. Risk prediction of individual metabolic risk scores for esophageal cancer-specific mortality in all study patients

\begin{tabular}{|c|c|c|c|c|c|c|c|}
\hline MRS & $\begin{array}{l}\text { Num. of } \\
\text { patients }\end{array}$ & cHR & $95 \% \mathrm{CI}$ & $\mathbf{P}$ & aHR & $95 \% \mathrm{CI}$ & $\mathbf{P}^{*}$ \\
\hline$\leq-6$ & 104 & 0.82 & $0.55-1.22$ & 0.321 & 0.95 & $0.63-1.45$ & 0.820 \\
\hline-5 & 86 & 0.68 & $0.44-1.05$ & 0.083 & 0.58 & $0.37-0.91$ & 0.019 \\
\hline-4 & 103 & 0.91 & $0.62-1.33$ & 0.616 & 0.88 & $0.58-1.34$ & 0.555 \\
\hline-3 & 138 & 0.84 & $0.59-1.21$ & 0.355 & 0.93 & $0.64-1.35$ & 0.704 \\
\hline-2 & 180 & 0.94 & $0.68-1.30$ & 0.703 & 0.91 & $0.65-1.29$ & 0.615 \\
\hline-1 & 177 & 0.90 & $0.65-1.24$ & 0.514 & 0.88 & $0.62-1.25$ & 0.484 \\
\hline 0 & 213 & 1.00 & Reference & group & 1.00 & \multicolumn{2}{|c|}{ Reference group } \\
\hline 1 & 191 & 0.99 & $0.73-1.36$ & 0.968 & 1.00 & $0.71-1.40$ & 0.988 \\
\hline 2 & 200 & 0.93 & $0.67-1.28$ & 0.648 & 0.86 & $0.61-1.22$ & 0.401 \\
\hline 3 & 188 & 0.98 & $0.71-1.35$ & 0.884 & 0.95 & $0.68-1.34$ & 0.777 \\
\hline 4 & 170 & 1.18 & $0.86-1.62$ & 0.309 & 1.15 & $0.82-1.62$ & 0.415 \\
\hline 5 & 129 & 1.19 & $0.85-1.66$ & 0.320 & 1.01 & 0.701 .45 & 0.953 \\
\hline 6 & 89 & 1.15 & $0.77-1.70$ & 0.499 & 1.10 & $0.73-1.67$ & 0.648 \\
\hline 7 & 71 & 1.58 & $1.08-2.33$ & 0.020 & 1.38 & $0.92-2.07$ & 0.123 \\
\hline 8 & 70 & 1.60 & $1.08-2.36$ & 0.018 & 1.31 & $0.87-1.98$ & 0.191 \\
\hline 9 & 52 & 1.91 & $1.26-2.90$ & 0.002 & 1.60 & $1.04-2.46$ & 0.034 \\
\hline 10 & 37 & 2.41 & $1.56-3.72$ & $<0.001$ & 1.83 & $1.15-2.93$ & 0.011 \\
\hline 11 & 41 & 1.91 & $1.24-2.95$ & 0.004 & 1.46 & $0.91-2.33$ & 0.113 \\
\hline 12 & 78 & 2.14 & $1.48-3.11$ & $<0.001$ & 1.62 & $1.10-2.39$ & 0.015 \\
\hline 13 & 61 & 1.68 & $1.14-2.50$ & 0.009 & 1.36 & $0.9-2.06$ & 0.143 \\
\hline 14 & 59 & 2.15 & $1.45-3.19$ & $<0.001$ & 1.74 & $1.15-2.63$ & 0.009 \\
\hline$\geq 15$ & 100 & 2.08 & $1.47-2.93$ & $<0.001$ & 2.03 & $1.40-2.95$ & $<0.001$ \\
\hline
\end{tabular}

Abbreviations: MRS, metabolic risk score; $\mathrm{cHR}$, crude hazard ratio; aHR, adjusted hazard ratio; $95 \% \mathrm{CI}, 95 \%$ confidence interval. MRS ranges from -9 to 17 . * $\mathrm{P}$ was calculated after adjusting for age, sex, smoking, histological differentiation, esophagus location, tumor embolus, tumor size, regional lymph node metastasis, subtype of esophageal cancer and tumour node metastasis stage.

Table 3. Risk prediction for esophageal cancer-specific mortality upon the tertile comparisons of metabolic risk score

\begin{tabular}{lllll}
\hline Group & Comparison* & HR & 95\% CI & $\mathbf{P}^{* *}$ \\
\hline Derivation & T2 vs. T1 & 1.43 & $1.17-1.75$ & $<0.001$ \\
& T3 vs. T1 & 2.28 & $1.90-2.73$ & $<0.001$ \\
Validation & T2 vs. T1 & 1.35 & $1.05-1.74$ & 0.020 \\
& T3 vs. T1 & 2.11 & $1.66-2.67$ & $<0.001$ \\
Combined & T2 vs. T1 & 1.22 & $1.04-1.43$ & 0.016 \\
& T3 vs. T1 & 2.37 & $1.95-2.88$ & $<0.001$ \\
\hline
\end{tabular}

Abbreviations: HR, hazard ratio; 95\% CI, 95\% confidence interval. * $\mathrm{T} 1$ : metabolic risk score $\leq 2$ (low); T2: metabolic risk score $>2 \& \leq 6$ (middle); T3: metabolic risk score $>6$ (high). ${ }^{* *} \mathrm{P}$ was calculated after adjusting for age, sex, smoking, histological differentiation, esophagus location, tumor embolus, tumor size, regional lymph node metastasis, subtype of esophageal cancer and tumour node metastasis stage.

\section{Risk stratification and re-stratification}

ROC analysis revealed that Harrell C-statistic for the model with TNM stage alone was 0.721 , which was increased to 0.743 with the addition of derived MRS in derivation group, and this increase was from 0.732 to 0.753 in validation group (Table 4). There was significant improvement in area under the ROC curve after adding MRS to TNM stage in both derivation and validation groups.

Calibration performance for the addition of derived MRS to TNM stage was evaluated by likelihood ratio test, as well as by AIC and BIC (Table 4). The significance of this test and the improvement in AIC and BIC, which measured the relative quality of a statistical model, indicated that MRS was indeed a part of the true model and carried a better fit.

\section{Discussion}

As an essential supplement to our recent investigations on single metabolic elements $[9,14]$, we, in this current study, borrowed successful experience of the Framingham heart study [19], and constituted MRS based on five baseline metabolism-related factors among 2535 EC patients from the ongoing FIESTA study [9-15]. Our findings supported the usefulness of baseline MRS in predicting the prognosis of postsurgical EC-specific mortality, underscoring the importance of monitoring metabolic risk factors before the surgery and the usefulness of MRS for risk stratification and therapeutic decision making.

Some studies have shown that metabolic syndrome represents a high-risk state triggering the development of cancer at many sites, including the esophagus [25-27]. During the latest decade, the prevalence of metabolic syndrome is continuously on the rise worldwide, and in mainland China, a large national survey in 2010 documented that the prevalence of metabolic syndrome was 33.9\% [28]. It is widely recognized that insulin resistance and hyperinsulinemia are hallmarks of metabolic syndrome, which is clinically featured by a collection of symptoms that can lead to heart disease, diabetes and cancer [29-31]. In return, growing data have supported the association of cancer at many sites with some symptoms of metabolic syndrome [32,33]. Although the exact mechanism linking metabolic syndrome to cancer thus far remains largely unknown, a working hypothesis is proposed about insulin resistance and insulin-like growth factor 1 system, mainly involving the contributions of adipokines, free fatty acids and aromatase activity [34]. Undoubtedly, the relationship between metabolic syndrome and EC needs additional investigations, such as from the aspect of clinical epidemiology.

In a large-scale prospective cohort study based on three European countries (the Me-Can project), metabolic syndrome was found to be associated with the risk of EAC, but not ESCC, and this association was largely mediated by BMI, an index of general obesity [35]. In an older population from the SEER-Medicare database, the presence of metabolic syndrome can account for increased EAC risk in males free of GERD and females regardless of GERD status [25]. However, existing data on the prognosis of metabolic syndrome for postsurgical EC are very limited, including only two studies from China $[14,36]$. One study by Wen and colleagues 

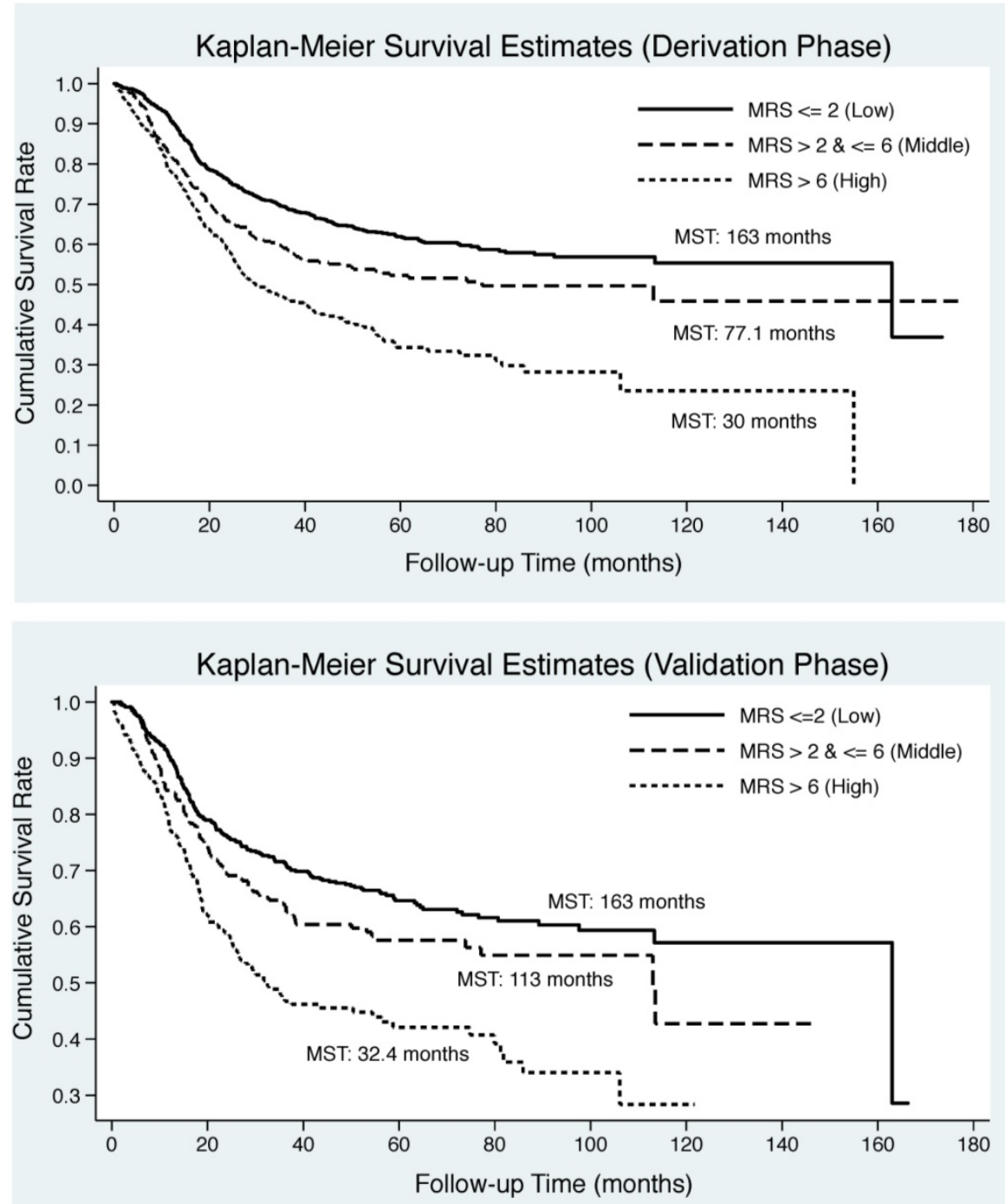

Figure 2. Kaplan-Meier curves by the tertiles of derived metabolic risk score for esophageal cancer-specific mortality in derivation (the upper panel) and validation (the lower panel) groups. Abbreviations: MRS, metabolic risk score; MST, median survival time.

retrospectively reviewed survival of 596 EC patients by metabolic syndrome, and they surprisingly found that metabolic syndrome was associated with a better prognosis after the surgery and improved histological differentiation [36]. The other was our recent FIESTA study [14], which indicated that baseline metabolic syndrome was an independent risk predictor for a poor prognosis of postsurgical ESCC males, to which hyperglycemia and dyslipidemia contributed remarkably. Our findings are of clinical importance, while understanding how these metabolism-related factors act at an individual or population level will represent a challenging paradigm for application of metabolic syndrome to EC-specific morality, which further motivates the implementation of this present study to quantify the magnitude of EC-specific mortality risk conferred by the accumulation of metabolic symptoms.
Table 4. Comparison of risk prediction models including TNM stage with and without metabolic risk score in both derivation and validation groups

\begin{tabular}{|c|c|c|c|c|}
\hline & \multicolumn{2}{|c|}{ Derivation group } & \multicolumn{2}{|c|}{ Validation group } \\
\hline & TNM stage & $\begin{array}{l}\text { TNM stage + } \\
\text { MRS }\end{array}$ & TNM stage & $\begin{array}{l}\text { TNM stage + } \\
\text { MRS }\end{array}$ \\
\hline \multicolumn{5}{|l|}{ Discrimination } \\
\hline C-statistic & 0.721 & 0.743 & 0.732 & 0.753 \\
\hline $\operatorname{AUC}(95 \% \mathrm{CI})$ & $\begin{array}{l}0.722(0.696, \\
0.748)\end{array}$ & $\begin{array}{l}0.736(0.710 \\
0.762)\end{array}$ & $\begin{array}{l}0.737(0.705, \\
0.769)\end{array}$ & $\begin{array}{l}0.751(0.719 \\
0.782)\end{array}$ \\
\hline $\begin{array}{l}\text { ROC curve } \\
\text { comparison }\end{array}$ & \multirow{2}{*}{\multicolumn{2}{|c|}{0.0125}} & \multicolumn{2}{|l|}{0.0210} \\
\hline \multicolumn{3}{|l|}{ Calibration } & & \\
\hline $\begin{array}{l}\text { Likelihood ratio } \\
\text { test }\end{array}$ & \multicolumn{2}{|c|}{ Chisq: $45.52(\mathrm{P}<0.0001)$} & \multicolumn{2}{|c|}{ Chisq: $26.24(\mathrm{P}<0.0001)$} \\
\hline AIC & 3169.57 & 3126.05 & 1975.27 & 1951.04 \\
\hline BIC & 3227.11 & 3188.82 & 2028.15 & 2008.72 \\
\hline
\end{tabular}


There are various modelling means in assessing the development of common complex diseases by synthesizing multivariate data for clinical use, such as the Framingham heart study 'points' system [19], survival-rate-based score system [37], standardized Z-score system [38] and so on. Among them, the Framingham heart study 'points' system is widely accepted as the most popular methodology for developing multivariable models to estimate the risk of cardiovascular disease [39]. We here drew lessons from this 'points' system and modified scores using the shrinkage method, to construct MRS based on five metabolism-related factors. To appraise the performance and clinical usefulness of this score, we generated MRS in derivation group, and justified its predictive utility in validation group. More importantly, incorporation of derived MRS can significantly improve discriminatory ability over the model containing traditional clinicopathologic parameters including TNM stage for postsurgical EC-specific mortality. Additionally, there was a great improvement in classification accuracy of traditional clinicopathologic model that incorporated MRS in both derivation and validation groups, reinforcing the appropriateness of the Framingham 'points' score system. However, as both derivation and validation groups were enrolled from the FIESTA study, and the generalizability of derived MRS to another population is limited. We agree that it would be a critical next step, with the aid of collaborative efforts and international consortia, to further externally validate the utility and usefulness of this derived MRS, with the ultimate goal of facilitating clinical decisionmaking and being applicable to individualized or precise medicine.

There are some limitations to this study. The main limitation may be the recruitment of study patients from a single cancer hospital consecutively from 2000 to 2010, and our findings could be better generalized if they can be repeated in other independent populations. Also, the present findings cannot be directly extrapolated to the general population, as only patients who received the surgery for EC were recruited. Moreover, the 5-year survival rate of these patients reached $52.2 \%$, which was exceedingly higher than the general population. In addition, data on anti-metabolic syndrome agents were only available for a very small proportion of study patients, and failure to adjust for drug regimens tended to bias risk prediction. Furthermore, only baseline metabolism-related factors were assessed, and frequently monitoring of these factors and analyzing their dynamic changes may provide valuable information on the prognostic appraisal of EC patients.
Taken together, extending our previous findings on metabolic syndrome and ESCC prognosis in the FIESTA study, we provided supportive evidence for the usefulness of baseline MRS in predicting the prognosis of postsurgical EC-specific mortality. This MRS can not only improve classification accuracy, but also improve discriminatory ability over the model containing traditional clinicopathologic parameters including TNM stage. From a medical prospective, it seems warranted for future well-designed prospective investigations to confirm the usefulness of this derived MRS for translational application in routine clinical practice and to decipher underlying mechanisms of metabolism-related factors in affecting the prognosis of EC patients.

\section{Supplementary Material}

Supplementary figures and tables.

http://www.jcancer.org/v09p1173s1.pdf

\section{Acknowledgements}

We thank our colleagues over the years at the Fujian Cancer Hospital - particularly Xiaohui Chen, Yuzhen Zheng, Qingfeng Zheng, Shuoyan Liu, Zhilian She, Kunshou Zhu, Weidong Zang, Weizhong Ruan, Weimin Fang, Lin Li, Mingqiang Chen, Derong Zhang, Shaofeng Lin, Shunjin Chen, Yigui Chen and Guohong Zhao for performing the surgery, Yanni Gao, Zhenzhou Xiao, Su Lin, Xuehong Liao, Wenhui Jiang, Jieqiong Lin, Xinjing Li, Yi Shi, Xiaojiang Wang, Shanfeng Jin, Hongfei Wang, Wucheng Shen, Weifeng Zhu, Xiaowen Cai, Baozhen Chen, Tongmei Chen, Xueyan Chen and Lifang Chen for collecting the blood/tissue samples and performing the follow-up investigations.

\section{Funding}

This study was financially supported by the Natural Science Foundation of Fujian Province (grants no. 2016J01503, 2016J01508) and the Training Project for Young and Middle-Aged Core Talents of Health System of Fujian Province (grants no. 2015-ZQN-JC-7, 2015-CX-7) and the National Clinical Key Specialty Construction Program of China (grant no. 2013-544).

\section{Specific author contributions}

$\mathrm{FP}, \mathrm{XZ}, \mathrm{DH}, \mathrm{WN}$ planned and designed the study, and directed its implementation; FP, DH, JL, $\mathrm{XZ}$ drafted the protocol; YC, XL, GC, BL, CL, HZ, YX obtained statutory and ethics approvals; $\mathrm{DH}, \mathrm{XL}, \mathrm{GC}$, $\mathrm{BL}, \mathrm{CL}, \mathrm{HZ}, \mathrm{YX}$ contributed to data acquisition; FP, $\mathrm{DH}, \mathrm{XZ}, \mathrm{WN}$ had access to all raw data; FP, DH, HS, SW, GX, GF, WN did the data preparation, quality control and data analysis; $\mathrm{WN}, \mathrm{FP}, \mathrm{DH}$ wrote the manuscript. 


\section{Availability of data and materials}

The datasets used and/or analyzed during the current study are available from the corresponding author on reasonable request.

\section{Competing Interests} interests.

The authors declare that they have no competing

\section{References}

1. Alexandre L, Long E, Beales IL. Pathophysiological mechanisms linking obesity and esophageal adenocarcinoma. World J Gastrointest Pathophysiol. 2014; 5: 534-49.

2. Dores GM, Qubaiah O, Mody A, Ghabach B, Devesa SS. A population-based study of incidence and patient survival of small cell carcinoma in the United States, 1992-2010. BMC Cancer. 2015; 15: 185.

3. Lin Y, Totsuka Y, He Y, Kikuchi S, Qiao Y, Ueda J, et al. Epidemiology of esophageal cancer in Japan and China. J Epidemiol. 2013; 23: 233-42.

4. Zeng H, Zheng R, Guo Y, Zhang S, Zou X, Wang N, et al. Cancer survival in China, 2003-2005: a population-based study. Int J Cancer. 2015; 136: 1921-30.

5. Tseng RC, Chang JM, Chen JH, Huang WR, Tang YA, Kuo IY, et al. Deregulation of SLIT2-mediated Cdc42 activity is associated with esophageal cancer metastasis and poor prognosis. J Thorac Oncol. 2015; 10: 189-98.

6. Mendonca FM, de Sousa FR, Barbosa AL, Martins SC, Araujo RL, Soares R, et al. Metabolic syndrome and risk of cancer: which link? Metabolism. 2015; 64: 182-9.

7. Pourhoseingholi MA, Ashtari S, Hajizadeh N, Zali MR. Metabolic Syndrome, Gastric Cancer Mortality and Competing Risk Survival Analysis. EBioMedicine. 2017; 15: 4-5.

8. You J, Liu WY, Zhu GQ, Wang OC, Ma RM, Huang GQ, et al. Metabolic syndrome contributes to an increased recurrence risk of non-metastatic colorectal cancer. Oncotarget. 2015; 6: 19880-90.

9. Hu D, Peng F, Lin X, Chen G, Liang B, Li C, et al. The elevated preoperative fasting blood glucose predicts a poor prognosis in patients with esophageal squamous cell carcinoma: The Fujian prospective investigation of cancer (FIESTA) study. Oncotarget. 2016; 7: 65247-56.

10. Hu D, Lin X, Chen Y, Chang Q, Chen G, Li C, et al. Preoperative blood-routine markers and prognosis of esophageal squamous cell carcinoma: The Fujian prospective investigation of cancer (FIESTA) study. Oncotarget. 2017; 8: 23841-50.

11. Peng F, Hu D, Lin X, Chen G, Li C, Chen Y, et al. The monocyte to red blood cell count ratio is a strong predictor of postoperative survival in colorectal cancer patients: The Fujian prospective investigation of cancer (FIESTA) study. J Cancer. 2017; 8: 967-75.

12. Hu D, Zhang H, Lin $X$, Chen G, Li C, Liang B, et al. Elevated preoperative neutrophil-to-lymphocyte ratio can predict poor survival in early stage gastric cancer patients receiving radical gastrectomy: The Fujian prospective investigation of cancer (FIESTA) study. J Cancer. 2017; 8: 1214-22.

13. Peng F, Hu D, Lin X, Chen G, Liang B, Zhang H, et al. Preoperative metabolic syndrome and prognosis after radical resection for colorectal cancer: The Fujian prospective investigation of cancer (FIESTA) study. Int J Cancer. 2016; 139: 2705-13.

14. Peng F, Hu D, Lin X, Chen G, Liang B, Zhang H, et al. Analysis of Preoperative Metabolic Risk Factors Affecting the Prognosis of Patients with Esophageal Squamous Cell Carcinoma: The Fujian Prospective Investigation of Cancer (FIESTA) Study. EBioMedicine. 2017; 16: 115-23.

15. Hu D, Peng F, Lin X, Chen G, Zhang H, Liang B, et al. Preoperative Metabolic Syndrome Is Predictive of Significant Gastric Cancer Mortality after Gastrectomy: The Fujian Prospective Investigation of Cancer (FIESTA) Study. EBioMedicine. 2017; 15: 73-80.

16. D'Agostino RB, Sr., Vasan RS, Pencina MJ, Wolf PA, Cobain M, Massaro JM, et al. General cardiovascular risk profile for use in primary care: the Framingham Heart Study. Circulation. 2008; 117: 743-53.

17. Edge SB, Compton CC. The American Joint Committee on Cancer: the 7th edition of the AJCC cancer staging manual and the future of TNM. Ann Surg Oncol. 2010; 17: 1471-4

18. Association C. The Suggestion on Chinese Metabolic Syndrome. Chinese Medical Association, Shanghai, China. 2004.

19. Sullivan LM, Massaro JM, D'Agostino RB, Sr. Presentation of multivariate data for clinical use: The Framingham Study risk score functions. Stat Med. 2004; 23: 1631-60.

20. Copas JB. Using regression models for prediction: shrinkage and regression to the mean. Stat Methods Med Res. 1997; 6: 167-83.

21. Kang CH, Kim YT, Jeon SH, Sung SW, Kim JH. Lymphadenectomy extent is closely related to long-term survival in esophageal cancer. Eur J Cardiothorac Surg. 2007; 31: 154-60.

22. Nishimaki T, Suzuki T, Kanda T, Obinata I, Komukai S, Hatakeyama K. Extended radical esophagectomy for superficially invasive carcinoma of the esophagus. Surgery. 1999; 125: 142-7.
23. Breitenstein S, DeOliveira ML, Raptis DA, Slankamenac K, Kambakamba P, Nerl J, et al. Novel and simple preoperative score predicting complications after liver resection in noncirrhotic patients. Ann Surg. 2010; 252: 726-34.

24. Vickers AJ, Elkin EB. Decision curve analysis: a novel method for evaluating prediction models. Med Decis Making. 2006; 26: 565-74.

25. Drahos J, Ricker W, Pfeiffer RM, Cook MB. Metabolic syndrome and risk of esophageal adenocarcinoma in elderly patients in the United States: An analysis of SEER-Medicare data. Cancer. 2017; 123: 657-65

26. Lin Y, Ness-Jensen E, Hveem K, Lagergren J, Lu Y. Metabolic syndrome and esophageal and gastric cancer. Cancer Causes Control. 2015; 26: 1825-34.

27. Drahos J, Li L, Jick SS, Cook MB. Metabolic syndrome in relation to Barrett's esophagus and esophageal adenocarcinoma: Results from a large population-based case-control study in the Clinical Practice Research Datalink. Cancer Epidemiol. 2016; 42: 9-14.

28. Lu J, Wang L, Li M, Xu Y, Jiang Y, Wang W, et al. Metabolic Syndrome among Adults in China - The 2010 China Noncommunicable Disease Surveillance. J Clin Endocrinol Metab. 2016: jc20162477.

29. Ehrhardt MJ, Mulrooney DA. Metabolic syndrome in adult survivors of childhood cancer: the intersection of oncology, endocrinology, and cardiology. Lancet Diabetes Endocrinol. 2015; 3: 494-6.

30. Micucci C, Valli D, Matacchione G, Catalano A. Current perspectives between metabolic syndrome and cancer. Oncotarget. 2016; 7: 38959-72.

31. Laakso M, Kuusisto J, Stancakova A, Kuulasmaa T, Pajukanta P, Lusis AJ, et al. The Metabolic Syndrome in Men study: a resource for studies of metabolic and cardiovascular diseases. J Lipid Res. 2017; 58: 481-93.

32. Bhindi B, Xie WY, Kulkarni GS, Hamilton RJ, Nesbitt M, Finelli A, et al. Influence of Metabolic Syndrome on Prostate Cancer Stage, Grade, and Overall Recurrence Risk in Men Undergoing Radical Prostatectomy. Urology. 2016; 93: 77-85.

33. Yang Y, Mauldin PD, Ebeling M, Hulsey TC, Liu B, Thomas MB, et al. Effect of metabolic syndrome and its components on recurrence and survival in colon cancer patients. Cancer. 2013; 119: 1512-20.

34. Uzunlulu M, Telci Caklili O, Oguz A. Association between Metabolic Syndrome and Cancer. Ann Nutr Metab. 2016; 68: 173-9.

35. Lindkvist B, Johansen D, Stocks T, Concin H, Bjorge T, Almquist M, et al. Metabolic risk factors for esophageal squamous cell carcinoma and adenocarcinoma: a prospective study of 580,000 subjects within the Me-Can project. BMC Cancer. 2014; 14: 103.

36. Wen YS, Huang C, Zhang X, Qin R, Lin P, Rong T, et al. Impact of metabolic syndrome on the survival of Chinese patients with resectable esophageal squamous cell carcinoma. Dis Esophagus. 2016; 29: 607-13.

37. Douglas S, Schild SE, Rades D. A new score predicting the survival of patients with spinal cord compression from myeloma. BMC Cancer. 2012; 12: 425.

38. Stocks T, Bjorge T, Ulmer H, Manjer J, Haggstrom C, Nagel G, et al. Metabolic risk score and cancer risk: pooled analysis of seven cohorts. Int J Epidemiol. 2015; 44: 1353-63.

39. Christophersen IE, Yin X, Larson MG, Lubitz SA, Magnani JW, McManus DD, et al. A comparison of the CHARGE-AF and the CHA2DS2-VASc risk scores for prediction of atrial fibrillation in the Framingham Heart Study. Am Heart J. 2016; 178: 45-54 